\title{
HABILIDADES SOCIALES Y ENFERMEDAD MENTAL
} Social skills and mental health disorders

\author{
Juan R. Pereira y José P. Espada*
}

\begin{abstract}
Resumen
El presente artículo se estructura en cuatro apartados, y analiza el concepto de habilidades sociales, un concepto en auge dentro de lo que se ha dado en llamar Psicología positiva. En primer lugar pretendemos delimitar el concepto, a continuación describimos la relación entre el déficit en habilidades sociales y la enfermedad. En un tercer apartado nos centramos en la relación entre la competencia social y la salud. Finalmente, en los últimos dos apartados analizamos la asociación existente entre las habilidades sociales y dos procesos concretos: la resiliencia y el éxito social.
\end{abstract}

Palabras clave: Habilidades sociales, enfermedad mental.

\begin{abstract}
This article is structured into four parts and analyzes the concept of social skills, a booming notion within the so-called Positive Psychology. First of all, this new notion will be defined. Then, the relationship between social skills deficit and mental health disorders will be explored, as well as the relationship between social competence and health. Finally, in the last two parts, the existing association between social skills and two concrete processes - resilience and social success - will be analyzed.
\end{abstract}

Key words: Social skills, mental health disorders.

\footnotetext{
* Universidad Miguel Hernández, Elche (España).
} 


\section{HABILIDADES SOCIALES: DEFINICIÓN Y DE- LIMITACIÓN DEL CONCEPTO}

El ser humano necesita de la sociedad para su crecimiento y desarrollo, en este sentido la sociedad es tanto un medio como un fin. Un medio en cuanto a que sería sumamente complicado, por no decir imposible, obtener los objetivos que nos proponemos si no es a través de la interacción con otras personas. Asimismo, se trata de un fin ya que, de por sí, las relaciones sociales son una fuente potencial de bienestar. Estaríamos hablando del doble objetivo de las habilidades sociales: objetivos instrumentales y objetivos afectivos.

Dentro del campo de estudio de la Psicología, el interés actual por las habilidades sociales y su implicación, tanto en el ámbito de la Salud como de la Enfermedad, es cada vez mayor. No obstante, el estudio psicológico de este tipo de habilidades tiene sus orígenes en la década de los años cincuenta o incluso antes. Salter (1949) ya hacía referencia a las habilidades expresivas (sobre todo expresión de acuerdo y desacuerdo), y con anterioridad, Murphy et al., (1937) describía la importancia de las habilidades relacionales en niños y jóvenes. Autores como Wolpe (1958) y Lazarus (1966) intentaron delimitar el concepto definiendo lo que por entonces se denominó conducta asertiva. Con el paso de los años, los investigadores han llegado a la conclusión de que las habilidades sociales no se limitan al uso de la asertividad. De hecho, las habilidades sociales exigen la puesta en marcha de una serie de habilidades en un entorno social complejo, algo que ya parecía señalar Philips (1961) cuando hace referencia a la competencia social.

Asimismo, al margen de la limitación teórica del concepto, muchos autores (Ellis, 1962, Moreno, 1978) han aplicado lo que hoy se conoce como entrenamiento en habilidades sociales observando lo práctico que suponía en la intervención clínica. Básicamente, se trata de enseñarle al individuo cómo llevar a cabo la conducta más beneficiosa para sus intereses sin que salga perjudicado a corto o largo plazo.

Básicamente, el origen del estudio de las habilidades sociales se encuentra en las Teorías del Aprendizaje Social, la Psicología Social y la Terapia de Conducta.
Según la Teoría del Aprendizaje Social (Bandura, 1982) la relación entre la persona y el ambiente está mediada por procesos de aprendizaje (procesos de modelado y moldeamiento) que pueden capacitar al sujeto para actuar de un modo socialmente competente. Esta teoría hace especial hincapié en la influencia del aprendizaje en la adquisición de un desempeño social competente. Esta perspectiva debe complementarse con la consideración de factores biológicos como el temperamento (inhibido o desinhibido). La Psicología Social enfatiza la importancia de la percepción, la atracción y la comunicación interpersonal. La Terapia de Conducta señaló aquellos comportamientos e intervenciones psicológicas relacionadas con el concepto de desempeño socialmente habilidoso. En este sentido, destacan las estrategias de role-playing, ensayo de conducta, refuerzo positivo y moldeamiento.

Con respecto a la definición de habilidad social, Caballo (1991) señala lo extremadamente complicado que es definir una conducta socialmente habilidosa. $\mathrm{Al}$ parecer, existen diferencias culturales, individuales y contextuales que hacen imposible la definición absoluta y universal de habilidad social.

Hablamos de diferencias culturales en cuanto a que lo que en una cultura puede ser visto como una puesta en marcha adecuada de relación en otra puede ser visto como todo lo contrario. Según un estudio comparativo del Instituto Max Planck para la Antropología Evolutiva en Leipzig (Alemania) las habilidades para la cognición social tales como entender las comunicaciones no verbales son propias del ser humano, siendo comunes a distintas culturas. Es decir, las habilidades en sí son algo propio del ser humano, pero el uso, las consecuencias sociales y la interpretación que se derive de este uso dependen de cada cultura. Las normas culturales marcan los patrones de comunicación adecuados e inadecuados en cada contexto y el uso esperable de dichas habilidades. Es lógico pensar que aquellas personas que sean capaces de adaptarse rápidamente a las normas de comunicación y relación propias de cada cultura hagan un mejor uso de las habilidades sociales.

El contexto en el que se mueva el sujeto también impide que se pueda establecer un criterio prefijado de lo que entendemos por habilidad social. El comportamiento esperable o socialmente aceptable está marca- 
do por modas, tendencias y situaciones sociales muchas veces cambiantes.

A nivel individual, variables sociodemográficas como la edad, el sexo o el nivel educativo, pueden jugar un papel fundamental como mediadoras en el uso de este tipo de habilidades. Además, como señala Caballo (1991) no existe un único uso correcto de las habilidades sociales, sino que distintos individuos, ejerciendo pautas comportamentales distintas, pueden conseguir sus objetivos siendo socialmente competentes.

Muchos autores (Bellack, 1985, Kelly, 1955) han hecho especial énfasis en las consecuencias como un factor determinante de lo que puede considerarse como conducta socialmente competente. La idea es que puede entenderse como socialmente habilidosa toda aquella expresión de emociones e intereses (negativos y positivos) sin que se pierda el refuerzo social. Sin embargo, a menudo, podemos encontrarnos con conductas patógenas que son reforzadas socialmente. En todo caso, es conveniente, como señala Linehan (1993), tener en cuenta tres tipos de eficacia interpersonal: eficacia del sujeto para lograr los objetivos, eficacia para mantener una buena relación y eficacia para mantener el autorrespeto.

Intentando llegar a una definición lo más adecuada posible, Caballo define las habilidades sociales como aquel: "conjunto de conductas realizadas por un individuo en un contexto interpersonal que expresa sentimientos, actitudes, deseos, opiniones o derechos de un modo adecuado a la situación, respetando esas conductas en los demás, y que generalmente resuelve los problemas inmediatos de la situación mientras reduce la probabilidad de que aparezcan futuros problemas" (p.407).

Según la definición anterior, el concepto de habilidades sociales incluye toda una serie de habilidades relacionadas. En el Cuadro 1 se sintetizan los principales términos relacionados con las habilidades sociales.

\section{Cuadro 1.}

\section{Conceptos relacionados con las habilidades sociales.}

Empatía: capacidad cognitiva de sentir en un contexto común lo que un individuo diferente puede percibir.

Asertividad: comportamiento comunicacional maduro en el que la persona ni agrede ni se somete a la voluntad de otras personas, sino que expresa sus convicciones y defiende sus derechos.

Resolución de problemas: proceso por el cual el individuo toman una decisión ante un conflicto valorando pros y contras, decidiéndose por la alternativa menos costosa y más beneficiosa.

Ya hemos señalado antes que las habilidades sociales no se limitan al clásico concepto de asertividad, sino que suponen una serie de comportamientos concretos. En un intento por operativizar el constructo, Caballo (1991) describe unas 13 dimensiones recogidas en la Tabla 1. No obstante, el que un individuo haga uso adecuado de una habilidad concreta no implica que haga el mismo uso o sea socialmente competente en otra dimensión, así pues, un sujeto puede ser socialmente competente en cuanto a hablar en público pero totalmente incapaz de disculparse o admitir su propia ignorancia.

Asimismo, un aspecto que frecuentemente está relacionado con el uso correcto de las habilidades sociales es el conjunto de conductas no verbales y paralinguísticas que rodean al comportamiento habilidoso. Particularmente importante es la mirada, la distancia interpersonal, la postura, los gestos, la expresión facial y la apariencia personal. Igual de importante es la correcta utilización de todos los componentes paralinguísticos como la entonación, el volumen de la voz, la claridad y la velocidad del habla.

Por su parte, Michelson (1987) señala que una conducta socialmente habilidosa se caracteriza por ser una habilidad adquirida por aprendizaje, que incluye comportamientos verbales y no verbales, que exige respuestas efectivas y apropiadas condicionadas por el medio. 


\section{Tabla 1}

\section{Dimensiones del constructo de Habilidades Sociales (Caballo, 1991).}

- Expresión de emociones.

- Expresión justificada de enfado o molestia.

- Aceptar cumplidos.

- Disculparse.

- Afrontamiento de las críticas

- Rechazar peticiones.

- Iniciar y mantener conversaciones.

- Hablar en público.

- Defensa de los propios derechos.

- Peticiones.

- Hacer cumplidos.

- Expresión de opiniones (aunque sean discordantes).

- Petición de cambio en la conducta del otro.

La evaluación de las habilidades personales es otro asunto complicado. El problema es que un concepto tan complejo y cargado de habilidades tan diversas siempre es difícil de operativizar. Al margen de los autoinformes y la observación, se han elaborado distintos instrumentos cuyo objetivo es recoger una medida de este constructo. Algunos instrumentos como el CEIC (Conocimiento de Estrategias de Interacción con los Compañeros, 1995) o el CEICA: (Entrevista sobre Estrategias de Interacción con los Compañeros para Adolescentes, 1995) de Díaz-Aguado y Royo son eminentemente prácticos. En el caso del CEIC por ejemplo, se trata de plantear al sujeto ocho historias que plantean problemas sociales, orientados a iniciar relaciones, ayudar a otro niño, resolver conflictos y conseguir objetivos. Otros cuestionarios estandarizados son: Batería de Socialización (BAS - 3) de Silva y Martorell (1987), Escala de Competencia Percibida (COPE) de Harter (1986), Batería de habilidades en la Solución de Problemas Interpersonales para el Ciclo Inicial de Pelechano (1991), Cuestionario de Educación Emocional (CEE) de GROP (Grupo de Investigación en Orientación Psicopedagógica, 1997) y Cuestionarios de Comunicación de Marroquín y Villa (1995): Cuestionario de Comunicación Interpersonal, Cuestionario de Comunicación Intrapersonal y Cuestionario de Pensamientos Distorsionados.

\section{HABILIDADES SOCIALES Y ENFERMEDAD MENTAL}

El déficit de habilidades sociales es tanto causa como consecuencia de enfermedad mental, entendiendo por enfermedad "una alteración de los procesos cognitivos y afectivos del desarrollo considerado como normal con respecto al grupo social de referencia del cual proviene el individuo" (OMS, 1946). En gran número de enfermedades mentales el área de las habilidades sociales está gravemente afectada. De hecho, el modelo de rehabilitación psicosocial preponderante en estos momentos (Liberman, 1993) establece como uno de los pilares de la rehabilitación el entrenamiento en habilidades sociales. Bien porque el sujeto no haya llegado a adquirir el aprendizaje de estas habilidades porque la enfermedad mental le ha sobrevenido demasiado pronto, como en el caso de la esquizofrenia de tipo hebefrénico, o bien porque las estrategias adquiridas sean altamente disfuncionales, como en determinados Trastornos de la Personalidad. Asimismo, al margen de que la enfermedad sea el origen principal del déficit, a menudo es el propio déficit el que deteriora todavía más al enfermo y cronifica su estado patológico, en determinados cuadros fóbicos, como por ejemplo el miedo a hablar en público, se produce un efecto de refuerzo mutuo entre el déficit de habilidades y la propia fobia. Es por esto, que muchos programas de efectividad comprobada incorporen de algún modo u otro el entrenamiento en habilidades sociales entre sus componentes; dependiendo del modelo de base de la Terapia se centrará en modificar cogniciones o conductas en relación al comportamiento del paciente frente a los demás (Tabla 2).

Como señala Linehan (1993) las terapias grupales son más útiles a la hora de trabajar la competencia social, dado que permiten la puesta en marcha de las habilidades durante el mismo proceso terapéutico de manera menos artificial que la que se suele dar en una terapia individual. Además en grupo puede fomentarse el aprendizaje por observación y se suele generar un compromiso público para el cambio, o al menos para la ejercitación de las habilidades entrenadas.

Por otro lado, el entrenamiento en habilidades sociales, no sólo es un componente fundamental de muchas terapias efectivas en población clínica, sino que también es una herramienta potente en términos de pre- 
vención de la enfermedad o conductas potencialmente peligrosas. Gran número de Programas de Prevención (del consumo de drogas o de conductas sexuales de riesgo, por ejemplo), así como gran número de programas de promoción de la Salud incluyen un módulo de entrenamiento en habilidades sociales. Mencionemos dos ejemplos característicos: el Programa Saluda de Prevención del Consumo de Alcohol y drogas de Síntesis (Espada y Méndez, 2003) y el Programa Prevenir el Sida (Espada, 2002). El Programa Saluda se basa en diversos aspectos preventivos cuya eficacia ha sido probada, el programa trabaja sobre la información, la refutación de los mitos publicitarios, la resolución de problemas, las alternativas al consumo y el entrenamiento en habilidades sociales. El Programa Prevenir el Sida, incorpora como un componente principal el entrenamiento en habilidades sociales. Se dedican varias sesiones a la mejora de la competencia social dirigida a la negociación con la pareja y la adopción de prácticas sexuales saludables.

Dependiendo de la conducta sobre la cual vamos a intervenir se hará más hincapié en algunas habilidades sociales frente a otras. En este sentido, en personas con algún tipo de adicción a tóxicos es fundamental el entrenamiento en habilidades de resistencia a la presión grupal, en sujetos con fobia social sería necesario el entrenamiento en habilidades de presentación, comunicación y aceptación de cumplidos, sujetos con problemas de control de impulsos se verían más beneficiados de un entrenamiento en empatía y afrontamiento de las críticas.

Un aspecto interesante es la distinción entre morbilidad oculta y morbilidad latente. Al margen de aquellos enfermos mentales que reciben atención, existe un gran número de personas que se verían beneficiadas de una intervención en entrenamiento en habilidades sociales a pesar de que sus limitaciones no le impidan llevar a cabo un funcionamiento más o menos adecuado, o a pesar de que su funcionamiento social no sea percibido por ellos como disfuncional a pesar de serlo. Este es el ejemplo del trastorno psicopático y de la delincuencia. De hecho, autores como Carkhuff (1971) señalan que el entrenamiento en habilidades sociales es el tratamiento más efectivo en la delincuencia. En el caso del tratamiento del trastorno psicopático de la personalidad las investigaciones no son tan esperanzadoras, al parecer la resocialización del psicópata es una empresa bastante complicada.

Tabla 2. Terapias eficaces en trastornos psicológicos graves que incorporan entrenamiento en algún tipo de habilidad interpersonal.

\begin{tabular}{|c|c|c|}
\hline Nombre de la intervención & Población diana & Componentes \\
\hline $\begin{array}{l}\text { Terapia Dialéctico } \\
\text { Conductual (Linehan, 1991) }\end{array}$ & $\begin{array}{l}\text { Trastorno de personalidad } \\
\text { Limite o Borderline. }\end{array}$ & $\begin{array}{l}\text {-Autoconciencia. } \\
\text {-Habilidades interpersonales. } \\
\text {-Estabilidad emocional. } \\
\text {-Control de impulsos. }\end{array}$ \\
\hline $\begin{array}{l}\text { Terapia Cognitiva de } \\
\text { los Trastornos de } \\
\text { personalidad (Beck, 1990) }\end{array}$ & $\begin{array}{l}\text { Trastornos de } \\
\text { personalidad del } \\
\text { Cluster A, B y C. }\end{array}$ & $\begin{array}{l}\text { Dependiendo del Trastorno: técnicas de } \\
\text { reatribución causal, reestructuración cognitiva, } \\
\text { asertividad, técnicas de escucha empática. }\end{array}$ \\
\hline $\begin{array}{l}\text { Terapia Racional- } \\
\text { Emotiva (Ellis, 1987) }\end{array}$ & $\begin{array}{l}\text { Trastornos del estado de } \\
\text { ánimo y ansiedad. }\end{array}$ & $\begin{array}{l}\text { Sustitución de pensamientos irracionales } \\
\text { por pensamientos racionales o emotivos } \\
\text { sobre uno mismo y los demás. }\end{array}$ \\
\hline $\begin{array}{l}\text { Terapia cognitivoestructural } \\
\text { (Guidano y Liotti, 1985) }\end{array}$ & $\begin{array}{l}\text { Trastornos del estado de } \\
\text { ánimo y ansiedad. }\end{array}$ & $\begin{array}{l}\text { Reestructuración cognitiva de las } \\
\text { estructuras mentales del paciente. }\end{array}$ \\
\hline $\begin{array}{l}\text { Terapia Psicológica } \\
\text { Integrada (Roder, 1996) }\end{array}$ & $\begin{array}{l}\text { Esquizofrenia y otros } \\
\text { trastornos psicóticos. }\end{array}$ & $\begin{array}{l}\text { Programa de Intervención grupal orientado } \\
\text { a la mejora de las habilidades del paciente } \\
\text { (habilidades sociales, de resolución de problemas } \\
\text { y de afrontamiento) y la mejora del apoyo familiar }\end{array}$ \\
\hline $\begin{array}{l}\text { Terapia de entrenamiento en } \\
\text { habilidades sociales }\end{array}$ & $\begin{array}{l}\text { Fobias y problemas } \\
\text { de ansiedad. }\end{array}$ & $\begin{array}{l}\text { Incluye técnicas de modelado, } \\
\text { autoinstrucciones y ensayo de conducta. }\end{array}$ \\
\hline
\end{tabular}


La fobia social es quizás el trastorno que mejor ejemplifica la incapacidad de poner en marcha las habilidades sociales y las consecuencias que de esto se derivan. Según el DSM-IV-TR (APA, 2004), los sujetos con fobia social muestran un temor acusado y persistente a las situaciones sociales, la exposición a situaciones sociales les provoca niveles altos de ansiedad, y suelen mostrar comportamientos de evitación. En este caso, vemos el resultado de la extrema afectación de la competencia social, ya que los pacientes con este diagnóstico ven afectada la casi totalidad de las habilidades sociales. Entre las consecuencias negativas de la fobia social destaca el bajo rendimiento académico o laboral, una reducción significativa de las relaciones sociales con la consiguiente pérdida de falta de red social de apoyo y alteraciones del estado de ánimo. El entrenamiento en habilidades sociales es una de las cuatro estrategias más efectivas, junto con las técnicas de contracondicionamiento, las técnicas de exposición y los tratamientos cognitivos conductuales. Al parecer, el déficit en habilidades sociales genera en los pacientes una falta de confianza en su desempeño a la hora de afrontar situaciones sociales (Overholser, 1990). Asimismo, y como hemos comentado anteriormente, la negativa a poner en práctica las habilidades sociales predispone a un mantenimiento de la fobia (Echeburúa, 2004). En todo caso, el entrenamiento en habilidades sociales parece mostrarse más efectivo cuando se aplica junto con otro tipo de estrategias, lo que obliga a llevar a cabo intervenciones multicomponentes.

Parece evidente que la mayoría de enfermedades mentales tienen afectada en mayor o menor medida la competencia social del paciente, en los trastornos por ansiedad y las alteraciones del estado de ánimo parece existir una incapacidad para poner en práctica una serie de habilidades sociales. En los trastornos psicóticos es posible que las habilidades sociales no se hayan llegado a adquirir, y en los trastornos de personalidad probablemente las habilidades aprendidas por los pacientes sean inadecuadas.

Como señala Liberman (1993) a menudo los sujetos con enfermedad mental no han adquirido competencia social o bien no han podido ponerlas en prácticas por diversos motivos. Por un lado es importante tener en cuenta la influencia de la familia y el entorno del sujeto, si los modelos del paciente no suponen un referente adecuado en lo que respecta a la competencia social quizás sea bastante complicado que el sujeto se beneficie del entrenamiento en este tipo de habilidades. Las terapias familiares de tipo sistémico (Shwartz, 1995) parecen ser una herramienta útil cuando la problemática familiar impide un desempeño adecuado de las habilidades sociales. Otro elemento que puede atrofiar el desempeño de las habilidades sociales es la institucionalización del enfermo mental, parece evidente que para hacer uso de la competencia social el paciente necesitará poder tener la oportunidad de ponerla en práctica.

Goldstein (1996) señala que una inadecuada competencia social suele ser un componente precedente en determinados trastornos mentales graves, según este autor, los niños preesquizofrénicos son menos expresivos emocionalmente y suelen mostrarse inseguros y socialmente ansiosos.

La terapia farmacológica es eficaz para la reducción de determinados síntomas a corto y medio plazo, pero si pretendemos buscar cambios a largo plazo debemos intervenir sobre la capacitación del sujeto para afrontar distintas situaciones, más allá de la situación puntual que de origen al problema. El entrenamiento en habilidades sociales proporciona al individuo una manera de amortiguar las situaciones estresantes ya que le permite tener herramientas de afrontamiento y solicitud de ayuda.

Como mencionábamos anteriormente, el entrenamiento en habilidades sociales no es tarea exclusiva de la persona con enfermedad mental, sino que es extensivo a sus modelos sociales de referencia y en su caso del terapeuta. De hecho, es difícil que un paciente comprenda la utilidad de este tipo de estrategias si quien pretende trasmitirle esta capacidad no es un referente adecuado. Por ello, es fundamental que el terapeuta haga un uso adecuado de su propia competencia social, mostrándose asertivo y empático (Millerr y Rollnick, 1991). 
Tabla 3

Capacidades del terapeuta en habilidades sociales (Liberman, 1993).

- Activo y colaborador.

- Capacidad para establecer objetivos concretos.

- Participativo e interactivo.

- Próximo.

- Alienta y da instrucciones.

- Ignora y suprime conductas perturbadoras.

- Empático.

- Asertivo.

- Ayuda al paciente a generar determinadas escenas y ensayos conductuales.

Las habilidades sociales están relacionadas con otro tipo de habilidades como el autocuidado o actividades básicas. De modo que presumiblemente, si un paciente tiene una alta competencia social, tendrá un mejor autocuidado, una mayor probabilidad de integrarse en actividades comunitarias y un mejor manejo de sus emociones.

\section{ENTRENAMIENTO EN HABILIDADES SOCIA- LES Y SALUD}

La OMS define la salud como el "estado de completo bienestar físico, mental y social, y no sólo la ausencia de enfermedad". A partir de esta definición podemos considerar la salud como la consecuencia de mantener un buen equilibrio en tres áreas: la física, la mental y la social. La interrelación entre estos tres factores definiría el estado de salud del sujeto (Green, 1988). Un sujeto sano socialmente debería poder vivir de manera autónoma, con el menor número de limitaciones posibles, y manteniendo una relación adecuada con su entorno.

Figura 1. Dimensiones de la Salud según la definición de la Organización Mundial de la Salud

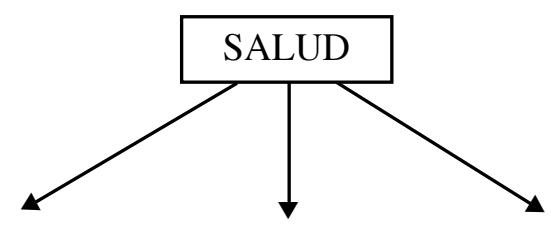

Biológica física

Psicológica mental
Social
La evolución histórica desde el Modelo Médico hacia el Modelo Psicosocial, ha supuesto que la salud se entienda como el resultado de los tres estados mencionados. Por lo que respecta al componente social, Asún (1993) señala que para que un individuo se encuentre en un estado socialmente sano debe ser capaz de interrelacionarse de manera satisfactoria con sus semejantes, debe conocer su entorno y participar en él de manera productiva y afrontarse de manera adecuada a los conflictos que se vayan planteando para conseguir una adaptación satisfactoria a su medio. En relación a esto, Johansen (1988) plantea que un sujeto sólo puede desarrollar sus capacidades sociales cuando su entorno se lo permita, para lo cual deberían asentarse las bases de una comunidad "sana":

- Vida social satisfactoria de los miembros que componen la comunidad. Los sujetos que componen la sociedad deberían mantener relaciones sociales que supusiesen cierto grado de satisfacción.

- Adecuado nivel de participación de los miembros que conforman la comunidad. Los sujetos deben tener la oportunidad de participar en su comunidad a través de diversos canales, de modo que se sientan identificados con esta.

- Capacidad de solicitar y utilizar los recursos necesarios para un buen funcionamiento social. En primer lugar deben existir dichos recursos y en segundo lugar deben estar disponibles para el uso de los miembros de la comunidad.

El Modelo Psicosocial reconoce la existencia de distintas necesidades en todo ser humano. Como señala Savary (1981), en el ser humano encontramos cuatro niveles de identidad (Figura 2). Estos cuatro niveles evolucionan continuamente y reciben influencias recíprocas unos de otros. Uno de estos niveles hace referencia al yo relacional, y está determinado por la relación que mantenemos con nuestros semejantes. Por lo que respecta a la necesidad relacional o construcción de un yo relacional, podríamos considerar diversas necesidades específicas:

- Seguridad relacional: las relaciones con los demás permite al sujeto sentirse acompañado y seguro ante situaciones aversivas. 
- Validación relacional: las relaciones permite validar y contrastar nuestras percepciones y emociones.

- Aceptación relacional: las relaciones supone una oportunidad de sentirse aceptado y reconocido.

- Impacto relacional: las relaciones permiten que generemos cambios en nuestro entorno.

- Expresión relacional: a través de las relaciones personales tenemos la oportunidad de expresar emociones y sentimientos.

Tabla 4

\begin{tabular}{ll}
\hline \multicolumn{2}{c}{ Niveles de identidad (Savary, 1981) } \\
\hline $\begin{array}{l}\text { Yo físico o biológico: } \\
\text { reacciones fisiológicas } \\
\text { y respuestas sensoriales. }\end{array}$ & $\begin{array}{l}\text { Yo relacional: conjunto de } \\
\text { relaciones mantenidas y roles } \\
\text { asumidos en las mismas. }\end{array}$ \\
$\begin{array}{l}\text { Yo psicológico o emocional: } \\
\text { emociones, pensamientos }\end{array}$ & $\begin{array}{l}\text { Yo íntimo: proyectos de futuro } \\
\text { y búsqueda de experiencias. }\end{array}$ \\
\hline
\end{tabular}

Estos postulados parecen indicar que existe relación entre el estado de salud y las relaciones sociales, y como consecuencia entre la salud y las habilidades de relación social de los sujetos. A nivel práctico, los resultados avalan los postulados del Modelo Biopsicosocial. Así por ejemplo, la investigación demuestra la existencia de relación entre un envejecimiento saludable y el mantenimiento de unas buenas relaciones sociales. El adecuado uso de las habilidades sociales es un predictor de la existencia de una red social de apoyo, lo cual influye de manera directa en el estado de salud de los ancianos. Aquellos ancianos con habilidades suficientes como para tener un confidente, mantener relaciones familiares y amistosas, y seguir siendo un miembro activo de la comunidad, presentan un mejor estado de salud.

La investigación sugiere que el cultivar unas relaciones interpersonales adecuadas se relaciona con el nivel de satisfacción personal de la población. Incluso a pesar de que el nivel socioeconómico sea considerablemente bajo (teniendo en cuenta comparativamente la renta per cápita de los países desarrollados). En este sentido, el equipo de investigación ESRC (Wellbeing in Devoloping Countries) de la universidad de Bath, en Inglaterra, llevó a cabo un estudio en Bangladesh. En esta investigación se evaluó el nivel de satisfacción general de distintas muestras poblacionales de las regiones más deprimidas de Bangladesh, concluyendo que el $79 \%$ de los encuestados se consideraban felices a pesar de sus bajos ingresos. El análisis posterior parece reflejar que el hecho de mantener una buena relación con la pareja y con otros miembros de la comunidad influye de manera decisiva en la calidad de vida de los encuestados.

En una investigación realizada por Argyle y Lu (1990) en el Departamento de Psicología Experimental de la Universidad de Oxford se analizó la relación entre las habilidades sociales y la felicidad. Al parecer la extraversión está relacionada con la felicidad, de modo que las personas extrovertidas refieren sentirse más satisfechas. Es probable que aquellas personas extrovertidas sean más competentes socialmente. Otros autores (Campbell, Converse y Rodgers, 1976) ya senalaron que diversas medidas de la competencia social eran buenos predictores de la felicidad de los sujetos.

Maslow (1968), señalaba que una vez cubiertas las necesidades fisiológicas y de seguridad, el ser humano buscaba satisfacer unas necesidades de amor o pertenencia. Se trata de la búsqueda de relaciones de pareja y amistad significativas, estables y enriquecedoras, así como el sentimiento de necesidad de pertenecer a una comunidad. Maslow aseguraba que aquellos sujetos autorrealizados (aquellos cuyo sentimiento de equilibrio y bienestar personal es muy elevado) se caracterizaban, entre otros aspectos, por una cualidad que él denominaba interés social o humanidad. Estas personas disfrutaban de relaciones personales íntimas y parecían mostrar una mayor habilidad para afrontar conflictos personales y desenvolverse con otras personas, resolviendo conflictos utilizando estrategias centradas en el problema, utilizando el sentido del humor y promoviendo la resolución en equipo de estos conflictos comunes. 
Figura 2

Pirámide de necesidades de Maslow (1968)

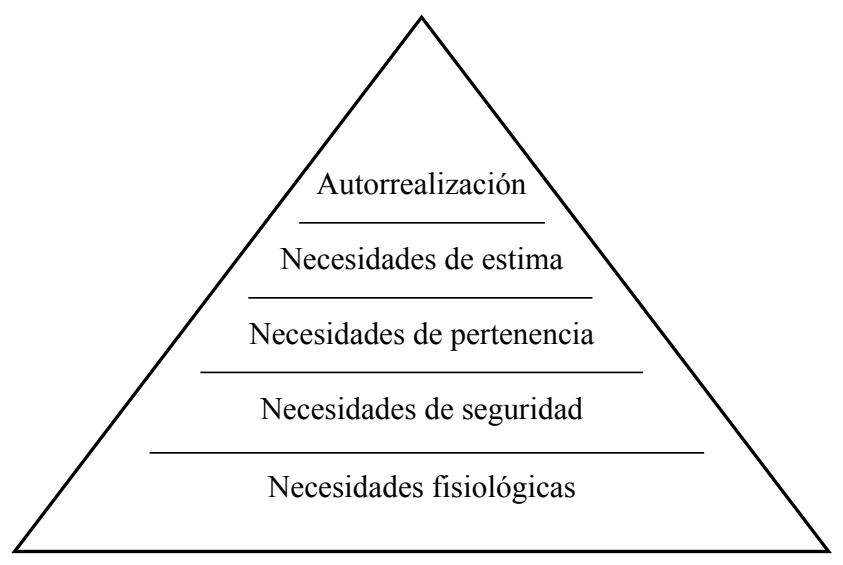

La escuela Humanista, más centrada en el análisis de la conducta y la cognición de sujetos considerados sanos y adaptados socialmente, hace especial hincapié en las relaciones sociales como factor determinante en el estado de bienestar del sujeto. Es un hecho que pasamos gran parte de nuestro tiempo en interacción con los demás, siendo la relación que mantenemos con nuestros semejantes fuente de bienestar o bien fuente de estrés. Dependiendo de las habilidades que pongamos en marcha podremos modular la relación que mantenemos con el entorno.

Un concepto que ha supuesto un avance en la consideración de las cualidades de las personas más satisfechas con sus vidas es el de Inteligencia emocional (Goleman, 1999). Según este autor, las personas mejor adaptadas y más satisfechas se caracterizan por ser inteligentes emocionalmente. Dentro de este concepto destaca la idea de inteligencia interpersonal, que consiste básicamente en realizar un uso adecuado de las habilidades interpersonales. Según este autor, la correcta utilización de las habilidades interpersonales supone un mayor nivel de bienestar psicológico y un mejor desarrollo de las relaciones con otras personas, fundamentalmente en tres áreas: familiar, social y profesional.

Dentro del interés por la Promoción de la Salud y la Educación para la Salud, el entrenamiento en habilidades sociales es especialmente relevante, ya que la competencia social no se considera como algo estático e inmutable, sino como algo sujeto al aprendizaje. Como en otros aspectos de la psicología, quitando los casos extremos de personas cuyas habilidades sociales son extremadamente sorprendentes o bien están totalmente limitadas, lo que nos queda es el grueso de la población normal. La mayoría de sujetos considerados normales y saludables hacen un uso de las habilidades sociales más o menos adecuado pero siempre mejorable. Desde la Psicología Positiva, el interés no es tanto el estudio de las personas excepcionales sino más bien el conocimiento de las herramientas que puedan mejorar sustancialmente a la mayor parte de la población.

Diener (1994) y Seligman (2002) afirman que el tener una vida social rica y satisfactoria es determinante en el nivel de bienestar subjetivo y felicidad. Asimismo, Buss (1989) indica que aquellos sujetos que establecen relaciones con conocidos basadas en la cooperación en lugar de en la competición muestran unos mayores niveles de bienestar emocional. La siguiente tabla refleja los componentes relacionales cuyo ejercicio aumenta el nivel de bienestar del sujeto según las investigaciones de Argyle (1992) y Diener (1994).

\section{Tabla 5. Estrategias relacionales que aumentan la sensación de bienestar.}

- Afrontamiento del conflicto a través de la cooperación.

- Mantenimiento de amistades íntimas.

- Relaciones frecuentes con la familia extensa.

- Saber perdonar los errores cometidos por los demás.

- Estilo comunicacional basado en la comunicación clara y educada.

Carr (2004) distingue la necesidad de tener en cuenta el apoyo social percibido, las relaciones de apoyo y las redes de apoyo como determinantes de la salud. Las personas con una percepción alta de apoyo social se muestran más eficaces obteniendo ayuda de los miembros de su red social cuando la necesitan y estructuran las situaciones de modo que se reduzca el estrés, lo cual reduce la sensación de malestar e invierte la posibilidad de que se cronifique una situación potencialmente patógena. Las relaciones de apoyo hacen referencia al afrontamiento de las situaciones difíciles y los retos vitales a los que inevitablemente todo ser humano debe hacer frente en algún momento de su vida. 
Las relaciones de apoyo son aquellas que se establecen con una persona significativa y que sirven de utilidad a la hora de afrontar situaciones complicadas desde el núcleo de poder formado por más de una persona, normalmente se trata de relaciones conyugales. Finalmente, con respecto a la red social, las personas con facilidad para mantener una red social amplia tienen mayor número de oportunidades para hacer frente a las situaciones conflictivas.

Según el Modelo Circumplejo de la conducta interpersonal (Wiggins y Trapnell, 1997) durante la infancia se establece un modo de interaccionar con los demás cuya función es la reducción de la inseguridad y la optimización de la autoestima. Según este modelo, cada sujeto establece un tipo concreto de interacción con los demás en base a dos ejes: dominación versus sumisión y hostilidad fría versus simpatía afectuosa. Los autores distinguen ocho tipos de patrones interpersonales distintos, siendo los patrones seguro/ dominante, gregario/extrovertido y simpático/afectuoso los que mayor relación tienen con el bienestar subjetivo.

\section{HABILIDADES SOCIALES Y ÉXITO}

Antes de profundizar en la relación entre las habilidades sociales y el éxito, debemos delimitar el concepto de éxito. Tener éxito está asociado a aquellas metas que nos marcamos individualmente, podríamos considerar que tenemos éxito cuando aquellos objetivos personales se ven cumplidos. Estos objetivos pueden ser de tipo académico, laboral o personal.

Conseguir nuestras metas implica cierto esfuerzo personal y la puesta en marcha de varias destrezas, entre ellas las destrezas sociales. Asimismo, el éxito está íntimamente relacionado con la valoración propia de cada sujeto y con la valoración que los demás hacen del comportamiento del mismo; es decir, lo que una persona puede considerar un éxito no tiene por qué ser considerado como tal por el resto de la sociedad. Un sujeto puede ser muy exitoso a nivel académico pero fracasar en su desarrollo cotidiano. Parece ser que la satisfacción vital no depende tanto del elevado éxito en un área como de los pequeños logros en varios ámbitos. En todo caso, la propia percepción de nuestros logros y la percepción que tengamos de lo que los de- más opinan de ellos influyen de manera decisiva en nuestra percepción de éxito o fracaso. Esto es algo sobre lo que Seligman (1991) ha hecho especial hincapié, distinguiendo entre estilos atribucionales internos o externos. En este sentido, las habilidades sociales hacen referencia a un atributo interno, de modo que aquellos sujetos que las ejerciten de manera correcta y sean concientes de ello tenderán a realizar atribuciones internas de éxito, lo cual supondrá una fuente añadida de bienestar. En este caso el sujeto se sentiría reforzado no sólo por haber conseguido sus objetivos, sino también por haberlos conseguido gracias a sus cualidades personales. Cuando el sujeto no cree en sus habilidades interpersonales, bien por una valoración errónea o por una limitación real, tenderá a fijarse objetivos sociales muy limitados o bien irreales, lo cual puede desembocar en un círculo vicioso basado en el refuerzo negativo.

Las habilidades interpersonales y el éxito están muy ligados a la idea de inteligencia práctica (Stenberg, 1985), inteligencia social (Ford y Maher, 1998), inteligencia interpersonal (Gardner, 1994) y más recientemente con el concepto de inteligencia emocional (Goleman, 2000). Aquellos sujetos con buenas habilidades interpersonales (una cualidad común de este tipo de inteligencias) tiene más probabilidades de tener una vida exitosa, entendido como un desarrollo cotidiano placentero (Goleman, 2000).

Estas definiciones de inteligencia hacen referencia a la habilidad para comportarse de manera respetuosa, eficaz y comprensiva con las personas que nos rodean. Las investigaciones de Cantor y Harlow (1994) revelan que aquellas personas más inteligentes socialmente son capaces de utilizar eficazmente procesos cognitivos como la memoria, la percepción y el razonamiento de modo que afrontan de manera más resolutiva las tareas sociales; asimismo, estas personas afrontan de manera más adecuada los momentos de transición vital, realizan planes de acción y buscan retroalimentación continua de sus progresos. Además, los sujetos habilidosos socialmente utilizan estrategias prácticas, centradas en el sentido común y la adaptación al entorno.

Stenberg (2000) analizó la diferencia entre sujetos exitosos y menos exitosos en su vida diaria, encontrando 
que aquellos sujetos más exitosos hacían uso de lo que él denominó conocimiento tácito, esto es la capacidad de adquirir conocimiento de manera inconciente a través del desempeño diario.

El conocimiento tácito implica la cualidad de aprender lo que funciona y lo que no casi de manera imperceptible. Estos sujetos más exitosos poseen un conocimiento adquirido a través de la resolución de problemas cotidianos, y aplican este mismo estilo de afrontamiento cuando emprenden objetivos personales, se trata de sujetos que dan más importancia a cómo hacer las cosas. Se trata, por ejemplo, de sujetos centrados en tener amistades en términos de calidad y no de cantidad.

Goleman (2000) señala que aquellos sujetos con mejores habilidades sociales, capaces de reconocer y manejar mejor sus emociones y las de los demás, están mejor posicionados a nivel vital. Una de las habilidades sociales más relevantes, según este autor, es la capacidad empática.

En el estudio de revisión bibliográfica llevado a cabo por Bar-On (1997) se llevó a cabo una recopilación de las características de personalidad que se habían asociado hasta el momento con el éxito. A raíz de este análisis se planteó un Modelo de cuatro dimensiones que componen la personalidad tendente el éxito, entre estos componentes encontramos las destrezas personales.

Tabla 6. Características de las personas exitosas.

$\begin{array}{ll}\text { Modelo de Goleman (2000). } & \text { Modelo de Bar-On (1997) } \\ \text { - Conocer las emociones propias. } & \text { - Habilidades interpersonales } \\ \text { - Controlar las propias emociones. } & \text { - Capacidad de adaptación. } \\ \text { - Automotivación. } & \text { - Manejo de la ansiedad. } \\ \text { - Identificar las emociones ajenas. } & \text { - Buen estado de ánimo. } \\ \text { - Manejar las relaciones. }\end{array}$

Como hemos mencionado al inicio de este apartado, el éxito es idiosincrásico de cada sujeto, de modo que cada persona puede considerar el éxito en función de sus objetivos y valores. Del mismo modo, cada persona tiene un modo distinto de proceder de cara a la consecución de sus objetivos. Sin embargo, los estu- dios indican que existen características comunes entre aquellas personas que alcanzan los objetivos que se plantean, Ketron y Bernstein (1981) señalan entre las cualidades comunes a los sujetos exitosos la resolución práctica de problemas, la habilidad verbal y la competencia social. La importancia de la competencia social parece mantenerse incluso entre distintos países (Yang y Stenberg, 1997).

Un concepto fundamental a la hora de abordar la relación entre el éxito y las habilidades interpersonales es la conducta de logro. Murray (1938) distinguió entre aquellas personas con alta y baja motivación de logro, se trata de la tendencia a vencer obstáculos y superar las tareas complejas lo antes posible. Distintos estudios (Veroff, 1983) relacionan la alta motivación de logro con el éxito laboral.

Otros concepto a tener en cuenta es el de autoeficacia (Bandura, 1986), se trata de la percepción personal acerca de la capacidad que un sujeto cree tener para conseguir una meta. Aquellos sujetos con una elevada motivación de logro y una alta autoeficacia tenderán a poner en marcha todos los recursos que vean a su alcance para conseguir sus objetivos, incluida la búsqueda de contactos que puedan suponer una ayuda.

En sus investigaciones sobre las diferencias de género y el éxito, Horner (1968) descubrió que cuando las mujeres hacían referencia al éxito femenino se centraban más en describir las consecuencias negativas que este éxito les pudiera suponer: impopularidad y marginación social. Sin embargo, cuando las mujeres hacían referencia al éxito masculino se centraban en las consecuencias positivas del mismo. En base a esto, la autora señaló la existencia de un "miedo al éxito" entre las mujeres, que según ella les bloqueaba la posibilidad de conseguirlo. No obstante, es probable que el éxito al que se hacía referencia en las investigaciones de Horner no tenga relación con las metas personales sino con lo socialmente esperable. Las diferencias encontradas sugieren que las mujeres valoran la popularidad y el éxito social por encima de los logros laborales o académicos. Este tema plantea un nuevo tema de debate: las diferencias de género en determinadas habilidades, en este caso habilidades interpersonales. Aunque es un tema discutible en el ámbito científico, y aunque las diferencias parece que tienden a difuminarse, 
las mujeres parecen mostrar una mayor capacidad para reconocer y manejar emociones y relacionarse a nivel interpersonal (Farris, 1969), lo cual es un predictor de un mejor ajuste y éxito social.

Según la Teoría del Intercambio (Thibaut y Kelley, 1959) los seres humanos mantenemos determinadas relaciones debido a una valoración de costes y beneficios. Las personas socialmente habilidosas reconocen rápidamente el punto crítico a partir del cual los costos son mayores a las recompensas y actúan buscando alternativas, ya sea modificando la relación que supone un costo o sustituyéndola. Se llevaría a cabo una comparación entre alternativas eligiendo la más adecuada.

La Teoría de la Equidad (Walsters, 1978) complementa la Teoría del Intercambio, y establece que las relaciones que establecemos buscan la equidad o reciprocidad entre las partes. Las investigaciones de Berscheid y Walster (1978) demuestran que aquellos matrimonios en los que existe una buena percepción de equidad, en los cuales los miembros persiguen objetivos similares, se muestran más satisfechos. Las personas hábiles socialmente suelen relacionarse con personas con las que comparten metas, de modo que las recompensas por el trabajo en equipo no sólo les beneficien a ellos. Estas personas son conscientes de que para conseguir el éxito individual es necesario contar con el apoyo de los demás.

Dentro de las habilidades interpersonales propias de las personas consideradas exitosas, destaca la empatía. Tanto Goleman (2000) como McClelland (1961) destacan la importancia de la empatía como determinante de las personas exitosas. Esta cualidad les permitiría no sólo sentir las emociones de los demás sino ver realmente la situación desde diversas perspectivas, ayudándoles a plantear de ese modo otras alternativas de intervención.

Según McClelland (1961) existen en el ser humano tres necesidades básicas: necesidad de logro, necesidad de poder y necesidad de afiliación. Las habilidades personales serían un elemento fundamental para cubrir cualquiera de las tres necesidades, sobre todo la necesidad de afiliación. McClelland reconoce que cada persona tiene una necesidad más imperante, sin embargo, las habilidades personales son necesarias para cubrirlas, ya que una persona con una necesidad de logro o poder fuerte puede tener problemas para satisfacerla si se muestra demasiado competitiva e inflexible. Es habitual que aquellas personas con una alta necesidad de logro sean demasiado exigentes con los demás y aquellas con una alta necesidad de poder sean demasiado inflexibles. Las personas con una alta necesidad de afiliación dan importancia a la relación interpersonal como fuente intrínseca de placer, y establecen relaciones basadas en la confianza y la comprensión mutua. Desde esta perspectiva vemos cómo la competencia social o la incompetencia social juegan un papel fundamental en el proceso de cubrir necesidades humanas.

Al margen de cuáles sean los objetivos que las personas se planteen (laborales, personales, colectivos, etc.) existen una serie de factores clave a la hora de conseguir el éxito. La credibilidad de las propuestas que se planteen para conseguir los objetivos parece ser un aspecto decisivo, ya que es mucho más probable conseguir metas cuando la propuesta de acción es creíble por parte de los demás (Mehrabian, 1972). Para conseguir credibilidad y confianza en las propuestas es necesario que las personas que las plantean sean comunicadores eficaces. La comunicación es un elemento básico de las habilidades interpersonales.

A modo de síntesis, debemos mencionar el modelo SPACE de Albrecht (2006). Este modelo pretende recoger las características de las personas exitosas socialmente. Albrecht distingue cinco dimensiones definitorias: empatía, claridad, autenticidad, presencia y sensibilidad social.

\section{Tabla 7. Modelo de Albrecht (2006)}

- Sensibilidad social o radar social: capacidad de comprender el entorno e interpretar el comportamiento de los individuos.

- Empatía: capacidad de comprender y conectar con los demás.

- Autenticidad: coherencia entre el comportamiento y los objetivos reales, es un factor determinante a la hora de generar confianza en los demás.

- Claridad: capacidad de trasmitir ideas de manera directa y transparente.

- Presencia: conjunto de gestos, posturas, conducta verbal y no verbal, comportamiento social. 


\section{HABILIDADES SOCIALES Y RESILIENCIA}

La resiliencia podría definirse como el fenómeno por el cual personas que se han enfrentado a un suceso o una serie de sucesos potencialmente traumáticos, consiguen sobreponerse y alcanzar una buena adaptación social y un equilibrio psicológico a pesar de lo que han vivido. Las situaciones traumáticas pueden ser de tipo agudo como el abandono, el duelo parental o una catástrofe natural, pero también pueden ser de carácter crónico: penurias económicas, abusos reiterados, etc. Por tanto, la resilencia no se limita a resistir una situación complicada, sino que implica la capacidad de rehacerse, de sobreponerse y superar esta situación. Sin embargo, el que un sujeto sea resiliente no quiere decir que sea invulnerable, como señalaba Rutter (1985): la resistencia al estrés es relativa, no absoluta (cualquier ser humano, por muy resiliente que sea, es susceptible de desmoronarse); además la resiliencia depende tanto de factores internos como sociales e institucionales, y varía con el tiempo en función de las circunstancias.

En psicología solemos hablar de factores de riesgo frente a factores de protección. Los factores de riesgo serían todos aquellos que predisponen a una persona a mostrarse vulnerable ante una situación complicada, los factores de protección hacen referencia a todos aquellos que protegen a la persona de desmoronarse ante el trauma. Masten y Coastworth (1998) distinguen entre ambientes familiares, individuales y ambientales. Entre los rasgos protectores internos encontramos las estrategias relacionales (empatía y capacidad de búsqueda de ayuda).

Tabla 8. Factores protectores y generadores de resiliencia según Masten y Coastworth (1998).

\begin{tabular}{lcc}
\hline Factores personales & Factores familiares & Factores sociales \\
\hline - Capacidad resolutiva. & - Buenas relaciones & - Apoyo en la escuela. \\
- Buenas estrategias de & familiares. & - Red social fuerte \\
coping. & - Apoyo del cónyuge. & fuera de la familia. \\
- Locus de control interno. & - Adecuada educación. - Participar en \\
- Buena autoeficacia. & & actividades culturales \\
- Buenas habilidades & y religiosas. \\
interpersonales. & \\
- Buena autoestima. & & \\
\hline
\end{tabular}

Cuando los factores familiares y sociales fallan, bien porque son el origen del problema, o bien porque no suponen un factor protector, el sujeto debe servirse de los factores personales para salir adelante.

Un aspecto que ha sido bastante estudiado es el análisis entre la relación de apego que se establece con la figuras de referencia materna y el estilo relacional del adulto resiliente. Aquellos jóvenes que han establecido relaciones de apego seguras tienden a mostrarse más coherentes en sus relaciones y dan más valor a las relaciones íntimas. Las relaciones de apego entre los hijos y sus padres se han visto notablemente alteradas en los últimos años debido a los cambios en la estructura familiar en la sociedad occidental actual, es el caso, por ejemplo, de las rupturas de pareja y el efecto que causan en los hijos (Orgilés, Espada y Piñero, 2007).

Mrazek y Mrazek (1987) elaboraron una lista con las cualidades de las personas que se mostraban resilientes:

- Optimismo y capacidad de esperar.

- Seguridad de ser querido y respetado por alguien.

- Comprensión y elaboración de las experiencias dolorosas.

- Altruismo.

- Capacidad para asumir riesgos.

- Capacidad de establecer lazos sociales y mantenerlos.

- Habilidad para conseguir información.

- Madurez.

- Capacidad de acondicionarse ante los peligros.

La capacidad de establecer lazos a la que los autores hacen referencia implica la habilidad del sujeto para relacionarse con otros, no sólo para recibir afecto, sino también para brindarlo, lo cual se entiende como una necesidad básica. Además, las habilidades sociales pueden estar presentes como mediadoras en otras cualidades resilientes como el altruismo o la habilidad para conseguir información.

Según los planteamientos de Kotliarenco, Cáceres y Álvarez (1996) basados en el Modelo de Grotberg (1997), existen cuatro fuentes potenciales de resiliencia. Estos autores distinguen cuatro yoes: "yo tengo" (fac- 
tores de soporte externo), "yo soy" (fuerzas internas), "yo puedo" (habilidades sociales) y "yo estoy" (lo que el sujeto está dispuesto a hacer). Vemos como estos investigadores dedican un módulo ("yo puedo") entero a las habilidades sociales.

Tabla 10. Modelo de Grotberg (1997) sobre la personalidad resiliente.

\section{Yo tengo (factores de soporte externo)}

- Personas de confianza y fuente de apoyo.

- Modelos sociales adecuados.

- Personas que fomentan la autonomía a través de un apego seguro.

\section{Yo puedo (Habilidades sociales)}

- Muestran un buen humor.

- Tienen buenas habilidades de comunicación.

- Buscan relaciones confiables.

- Establecen relaciones de ayuda (tanto para darla como para recibirla).

- Son creativos.

- Son persistentes.

\section{Yo soy (fuerzas internas)}

- Autoestima y autoconfianza.

- Habilidades de autoconciencia.

- Autorrespeto.

\section{Yo estoy (factores motivacionales)}

- Motivación por cambiar aquellas cosas que nos desagradan.

- Optimismo.

- Con confianza en el futuro.
Asimismo, parecen existir diferencias de género, siendo las mujeres resilientes quienes hacen más uso de estrategias basadas en las relaciones interpersonales frente a los hombres resilientes que suelen mostrarse más pragmáticos (Grotberg, 1997).

En todo caso, las personas resilientes responden mejor al contacto con sus semejantes, y generan emociones más favorables en los demás. Como hemos visto, se comunican más fácilmente, se suelen implicar en conductas prosociales y hacen un buen uso del sentido del humor.

Mussuto y Piracés (2007) remarcan el papel fundamental de las habilidades interpersonales en las personas resilientes. Según estos autores el aislamiento social es una situación nada común en las personas resilientes. El comunicarse con otras personas y mantener relaciones estrechas con ella les permite, a los resilientes, mantenerse apoyados en un ambiente aversivo. Las habilidades sociales les permiten aprender de las experiencias de los demás tanto como de las suyas.
Por otro lado, se suele hacer referencia al concepto de familias comunidades resilientes. La resiliencia a menudo surge de una interacción familiar y comunitaria adaptativa. Las relaciones interpersonales establecidas en un entorno resiliente tienen características propias: se establecen relaciones de confianza en el protagonista del cambio, a través de la conversación se llegan a acuerdos estratégicos sobre cómo actuar. Las habilidades interpersonales puestas en marcha por los sujetos y las familias resilientes implican la comunicación adecuada, la confianza, la cohesión y la puesta en común a la hora de actuar. Los sujetos resilientes suelen hacer uso de estrategias de coping para resolver problemas y enfrentarse a la toma de decisiones, de ahí la importancia del modelo familiar (Espada, Sánchez y Pereira, 2008)

Pero sin duda, la importancia de la resiliencia es que contradice el determinismo biológico que establecería que si alguien sufre de una serie de situaciones complicadas en su vida está abocado a la patología mental. De hecho, el fenómeno de la resiliencia no se limita a un grupo limitado de casos excepcionales de 
personas que han superado airosamente situaciones extremas, un grupo poblacional importante se ha enfrentado a situaciones críticas superándolas con un nivel más o menos alto de éxito. Asimismo, si las cualidades resilientes se pueden aprender, estaríamos ante un abanico de herramientas que nos permitirían entrenar a las personas en habilidades que servirían de amortiguadores ante situaciones difíciles. Entre estas habilidades podemos destacar la autoconciencia y las habilidades relacionales. Se trataría de preparar al individuo e incluso a la familia, en habilidades que les sirviesen para afrontar $\mathrm{u}$ futuro que se prevé complicado.

Un aspecto afín a la resiliencia es el de resistencia, la diferencia es que la resiliencia implica la habilidad de recomponerse tras resistir el impacto del suceso en cuestión. Resistir implica no dejar que lo ocurrido provoque un desajuste psicológico grave y crónico. Teniendo en cuenta que muchos de los sucesos a los que nos enfrentamos implican la interacción humana, parece evidente el papel básico de las habilidades interpersonales. Este es el caso, por ejemplo, de los abusos sexuales. Las habilidades de resistencia a la presión o el entrenamiento en percepción de señales verbales y no verbales resultan especialmente relevante en el caso de los malos tratos infantiles. Las familias en las cuales suele producirse una situación de abuso son familias poco cohesionadas, con una estructura caótica en la que se establecen constantes discusiones entre padres e hijos.

Cuando las situaciones estresantes no son agudas sino crónicas, el papel de las habilidades sociales también es importante. Las investigaciones demuestran que ante una situación de estrés continuo, el entrenamiento en relajación, el entrenamiento en habilidades interpersonales y resolución de conflictos y el entrenamiento en reestructuración cognitiva e inoculación del estrés suelen combinarse como tratamiento de elección.

Uno de los estudios sobre la personalidad resiliente más importante, es el de Werner (1989). Este investigador siguió a una cohorte de 700 niños pobres en una situación socioeconómica estresante. Este seguimiento a largo plazo identificó a un tercio del grupo inicial con características resilientes, a pesar de su grave situación habían superado las situaciones críticas, al entrar en la edad adulta tenían una buena adaptación so- cial y un buen desempeño en distintos ámbitos. Analizando las distintas características de este subgrupo de sujetos resilientes Werner identificó dos aspectos comunes a casi todos los sujetos que componían este subgrupo resilientes: la influencia ejercida por un vínculo significativo y la participación en la religión. Con respecto al vínculo significativo, los sujetos resilientes hacían referencia a la relación establecida con personas significativas que funcionaban como un tutor o guía ante la adversidad.

En general, una persona puede disponer de fuerzas intrapsíquicas y de habilidades interpersonales pero aún así no mostrarse resiliente, esto puede deberse a la falta de oportunidades para poner en práctica sus habilidades, la sociedad debe poner a disposición de las personas, y en concreto de aquellas personas más vulnerables, una serie de recursos que les permitan poner en práctica sus habilidades.

\section{REFERENCIAS}

Albrecht, K. (2006). Inteligencia social. Barcelona: Vergara.

American Psychiatric Association (2000). Diagnostic and Statistical Manual of Mental Disorders (DSM$I V$-TR). Washington, DC: Author

Argyle, M. (1992). La psicología de la felicidad. Madrid: Alianza Editorial.

Argyle, M. y Lu, L. (1990). Happiness and social skills. Personality and Individual Differences, vol. 11(12): 1255-1261.

Asún, D. (1993) Algunas reflexiones sobre psicología comunitaria. En: R. Olave \& L. Zambrano (Eds.). Psicología comunitaria y salud mental en Chile. Santiago de Chile, Universidad Diego Portales.

Bandura, A. (1986) Social foundation of thoughts and action: a social cognitive theory. Englewood Cliffs, Prentice-Hall.

Bandura, A y Walters, R (1982) Aprendizaje social y desarrollo de la personalidad. Madrid: Alianza.

Bar-On, R. (1997). BarOn Emotional Quotient Inventory. Technical Manual. Toronto: MultiHealth Systems. 
Beck, A, T. y Freeman,A. (1990). Terapia cognitiva de los trastornos de personalidad. Barcelona: Paidós.

Bellack, A. S y Hersen, M. (1985). Dictionary of Behavior Therapy Techniques. New York.: Pergamon.

Buss, H. (1989). Personality as traits. American Psychologist. 1378- 1388.

Caballo, V. E. (comp.) (1991). Manual de técnicas de modificación y terapia de conducta. Madrid: Siglo XXI.

Campbell, A., Converse, P. E. y Rodgers, W. L. (1976). The quality of American life: Perceptions, evaluations and satisfactions. New York: Russell Sage Foundation.

Cantor, N., y Harlow, R. E. (1994). Social intelligence and personality: Flexible life task pursuit. En: R. J. Sternberg, y P. Ruzgis (eds.), Personality and intelligence. New York: Cambridge University Press.

Carkhuff, R. R. (1971). The development of human resources. New York: Holt.

Carr, A. (2004). Positive Psychology: the science of happiness and human strengths. Routledge.

Díaz-Aguado, M. J. y Royo, P. (1995). La evaluación de la competencia socioemocionala través de una entrevista semiestructurada. En: M.J. Díaz-Aguado (Dir.). Niños con dificultades socioemocionales: Instrumentos de evaluación. Madrid: Ministerio de Asuntos Sociales.

Diener, E. (1994). El bienestar subjetivo. Intervención Psicosocial, 2(8), 67-113.

Dion, K., Berscheid, E. y Walster, E. (1972). What is beautiful is good. Journal of Personality and Social Psychology, 24, 285-290.

Echeburúa, E. (2004). Superar un trauma. El tratamiento de las víctimas de sucesos violentos. Madrid. Pirámide.

Ellis, A. y Dryden, W. (1987). Práctica de la Terapia Racional Emotiva. Bilbao: Desclée de Brouwer.

Espada, J. P. y Méndez, F. X. (2003). Programa SALUDA: Prevención del abuso del alcohol y del consumo de drogas de síntesis. Madrid: Pirámide.
Espada, J. P., Pereira, J. R. y García-Fernández, J. M. (2008). Influencia de los modelos sociales en el consumo de alcohol en adolescentes. Psicothema.

Espada, J. P. y Quiles, M. J. (2002). Prevenir el Sida. Madrid: Pirámide.

Ellis, A. (1962). Razón y Emoción en Psicoterapia. Bilbao: DDB.

Farris, G. F., y Lim, F. G., (1969). Effects of performance on leadership, cohesiveness, influence, satisfaction, and subsequent performance. Journal of Applied Psychology, issue 53.

Ford, M. E. y Maher, M. A. (1998). Self-awareness and social intelligence. In M.D. Ferrari \& R. Stenberg, Eds. Self-awareness: its nature and development (pp. 191-218). New York: Guilford Press.

Gardner, H. (1994). Educación artística y desarrollo humano. Barcelona: Paidós.

Goldstein, M. (1996). Psychoeducational family interventions in psychotic disorders. New Trends in Experimental and Clinical Psychiatry, 11:71-79.

Goleman, D. (1999). La Inteligencia Emocional en la Empresa. Buenos Aires: Vergara.

Goleman, D. (2000) Inteligencia emocional. Barcelona, Kairós.

Green, W. y Simons, B. (1988). Educación para la salud. México: McGraw - Hill.

Grotberg, E. (1997). Resiliencia. Descubriendo las propias fortalezas. Buenos Aires: Paidós

Guidano, V. y Liotti, G. (1985). Una base constructivista para la terapia cognitiva, En: Mahoney, Michael y Freeman, A. Cognición y psicoterapia. Barcelona: Paidós.

Harter, S. (1985). Manual for the self-perception profile for children. Colorado: University of Denver.

Horner, M.S. (1968). Sex differences in achievement and performance. Social learning and clinical psychology. New York: Prentince Hall.

Informe Wellbeing in Devoloping Countries (2008). Universidad de Bath, Inglaterra.

Johansen, O. (1988). La comunidad como sistema dinámico. Santiago de Chile: Editorial de Ciencias económicas y administrativas. 
Kelly, G. A. (1955). The Psychology of Personal Constructs. New York: Norton and Company.

Kotliarenco, M.A.; Cáceres, I. y Alvarez, C. (eds.) (1996). Resiliencia: construyendo en adversidad. En: Seminario Pobreza, Desarrollo Humano e Intervención Social: la Resiliencia como una Posibilidad, organizado por CEANIM, Santiago de Chile.

Lazarus, R. S. (1966). Psychological Stress and the Coping Process. New York: McGraw-Hill.

Liberman, R. (1993). Rehabilitación Integral del Enfermo Mental Crónico. Barcelona: Martinez Roca.

Linehan, M. (1993). Manual de tratamiento de los trastornos de personalidad límite. Paidós. Barcelona.

Marroquín, M. y Villa, A. (1995). La Comunicación Interpersonal. Medición y estrategias para su desarrollo. Bilbao: Mensajero

Maslow, A. (1968). Toward a Psychology of Being. New York: D. Van Nostrand.

Masten, A. y Coastsworth, J. (1998) El desarrollo de competencia en ambientes favorables y desfavorables: Lecciones en estudios sobre niños exitosos. Psicología Americana, 53(2), 205-220.

Mehrabian, A. (1972). Silent messages. Belmont, CA: Wadsworth.

McClelland, D. (1961). The achieving society. Princenton, New Jersey: Van Nostrand.

Michelson, L., Suga I. D. y Word, R. (1987). Las habilidades sociales en la infancia. Barcelona: Martínez Roca.

Miller, W. y Rollnick, S. (1991). Motivational Interviewing: Preparing People for Change in Addictive Behaviour. New York: Guilford Press.

Murphy, G., Murphy, L. B. y Newcomb, T. M. (1937). Experimental Social Psychology. New York: Harper and Row.

Murray, H. A. (1938). Explorations in personality. New York: Oxford University Press.

Mussuto, M. S. y Piracés, A. (dir.). (2007). Creencias adictivas y severidad de la adicción a la cocaína [CD ROM]. Mendoza: Facultad de PsicologíaUDA.
Moreno, J. L. (1978). Psicodrama. Buenos Aires. Hormé.

Mrazek P. J. y Mrazek, D. (1987). Resilience in child maltreatment victims: A conceptual exploration. Child Abuse. 11: 357-365

Organización Mundial de la Salud (1946). Conferencia internacional sobre la salud. Actas oficiales OMS. New York: OMS

Orgilés, M., Espada, J. P. y Piñero, J. (2007). Intervención psicológica con hijos de padres separados: experiencia de un punto de encuentro familiar. Anales de Psicología, 23(2) 240-244.

Overholser, J. C. y Fritz, G. K. (1990). The impact of childhood cancer on the family, Journal of Psychosocial Oncology, 8(4), 71-85.

Pelechano, V. (1995). Inteligencia social y habilidades interpersonales. Análisis y Modificación de Conducta,10 (26): 393-420.

Phillips, L. y Zigler, E. (1961): Social competence: The action-thought parameter and vicariousness in normal and pathological behaviors, Journal of Abnormal and Social Psychology, 63, 137-146.

Roder, V. (1996). Terapia integrada de la esquizofrenia. Madrid: Ariel.

Rutter, M. (1985). Psiquiatría del desarrollo. Barcelona: Salvat.

Salter, A. (1949). Condicioned Reflex Therapy. Farrar, Straus.

Savary, L. (1981). Como lograr una nueva personalidad. Buenos Aires: Ludium.

Seligman, M. (2002). La auténtica felicidad. Barcelona: Vergara.

Seligman, M. (1991). El optimismo se adquiere. Buenos Aires: Atlántida.

Silva, F. y Martorell, M. C. (1987). Cuestionario I-6 (Junior): Adaptación española. Evaluación Psicológica. Psychological Assessment, 3: 55-78.

Schwartz, R. C. (1995). International Family Systems Therapy. New York: The Guilford Press.

Stenberg, R. J. (1985). Beyond IQ: A triarchic theory of human intellegence. Nueva York: Cambridge University Press 
Stenberg, R. J. (2000). Inteligencia exitosa. Barcelona, Paidós.

Sternberg, R. J., Conway, B. E., Ketron, J. L., y Bernstein, M. (1981). People's conception Intelligence. Journal of Personality and Social Psychology, 41(1), 37-55

Thibaut, J. W. y Kelly, H. H. (1959). The Social Psychology of Groups. New York: John Wiley and Sons.

Veroff, J. (1983). Contextual determinants of personality. Personality and Social Psychology Bulletin, 9, 331-344.

Walster, E., y Walster, G. W. (1978). A new look at love. Reading, Mass: Addison-Wesley.

Walster, E., Walster, G. W., y Berscheid, A. (1978). Equity theory and research. Boston: Ally and Bacon.
Werner, E. E. (1989). High-risk children in young adulthood: a longitudinal study from birth to 32 years. American Journal of Orthopsychiatry, 59(1), pp. 72-81

Wiggins, J. S., Phillips, N. y Trapnell, P. (1989). Circular Reasoning About Interpersonal Behavior: Evidence Concerning Some Untested Assumptions Underlying Diagnostic Classification. Journal of Personality and Social Psychology, 56(2) 296-305.

Wolpe, J. (1958). Psychotherapy by reciprocal inhibition. Stanford: Stanford University Press.

Yang, S. y Stenberg, R. J. (1997). Conceptions of intelligence in ancient Chinese philosophy. Journal of theoretical and Philosophical Psychology, 17 (2), 101- 119.

Fecha de recepción: 15 de enero, 2010

Fecha de aceptación: 17 de marzo, 2010 